\title{
A frequentist and Bayesian regression analysis to daily peak electricity load forecasting in South Africa
}

\author{
D. Chikobvu ${ }^{1}$ and C. Sigauke ${ }^{2 *}$ \\ ${ }^{1}$ Department of Mathematical Statistics and Actuarial Science, University of the Free State, P. O. Box 339, \\ Bloemfontein, 9300, South Africa. \\ ${ }^{2}$ Department of Statistics and Operations Research, School of Mathematical and Computer Sciences, \\ University of Limpopo, Turfloop Campus, P. Bag X1106, Sovenga, 0727, South Africa.
}

Accepted 15 June, 2012

\begin{abstract}
A frequentist and Bayesian regression analysis to a piecewise linear regression model for daily peak electricity load forecasting in South Africa for the period 2000 to 2009 is discussed in this paper. The developed model captures a wide variety of electricity demand drivers such as temperature, seasonal, lagged demand and calendar effects. A Bayesian analysis provides a way of taking into account uncertainty in the estimation of the piecewise linear regression parameters. Uncertainty about the true values of the Bayesian parameter estimates is incorporated into the analysis through the use of a noninformative prior distribution. The results obtained are easy to explain to management. Empirical results showed that an increase in electricity peak demand, if temperature decreases by ${ }^{\mathrm{C}} \mathrm{C}$, could be any value between 140 and $200 \mathrm{MW}$ during the winter months. Similarly during the summer months the increase in electricity peak demand, if temperature increases by $1^{\circ} \mathrm{C}$, ranges from -20 to $80 \mathrm{MW}$. There is a persistent increase of around $2 \mathrm{MW}$ in hourly electricity peak demand with time in South Africa. Electricity demand in South Africa is more sensitive to the winter period. Demand for electricity during holidays decreases significantly compared to a day before and after a holiday. This information and the quantification of such uncertainty are important for load forecasters in the power Utility Company in South Africa (Eskom) as it helps them in the determination of consistent and reliable supply schedules.
\end{abstract}

Key words: Posterior distribution, temperature, piecewise linear regression, load forecasting.

\section{INTRODUCTION}

Short term electricity load forecasting is very important for system operators who have to ensure that the amount of electricity drawn from the grid and the amount generated balances (Cottet and Smith, 2003; Taylor, 2006). In the absence of blackouts and load-shedding the electricity load is equal to electricity demand. Load forecasting is generally divided into short-term, medium-term and longterm forecasting. Most papers in literature concentrate on short-term point forecasts (Munoz et al., 2010). Shortterm load forecasting is important to ensure that there is a balance between demand and supply since electricity cannot be stored (Munoz et al., 2010).

${ }^{*}$ Corresponding author. E-mail: csigauke@gmail.com.
Demand drivers of electricity are generally split into, economic factors, weather variables and calendar effects. In short-term forecasting weather variables such as temperature and calendar effects are usually incorporated in models for electricity demand. The influence of temperature on daily electricity load forecasting has been studied extensively in the energy sector using classical (frequentist) statistics time series, regression based methods including artificial neural networks (Fan and Hyndman, 2011; Hekkenberg et al., 2009; Mirasgedis et al., 2006; Saini, 2008).

Most papers in literature concentrate on point forecasting only. One major drawback of point forecasting only is that it does not take into account uncertainty in the estimation of the parameters. One way of overcoming this, is the use of Bayesian analysis and density 


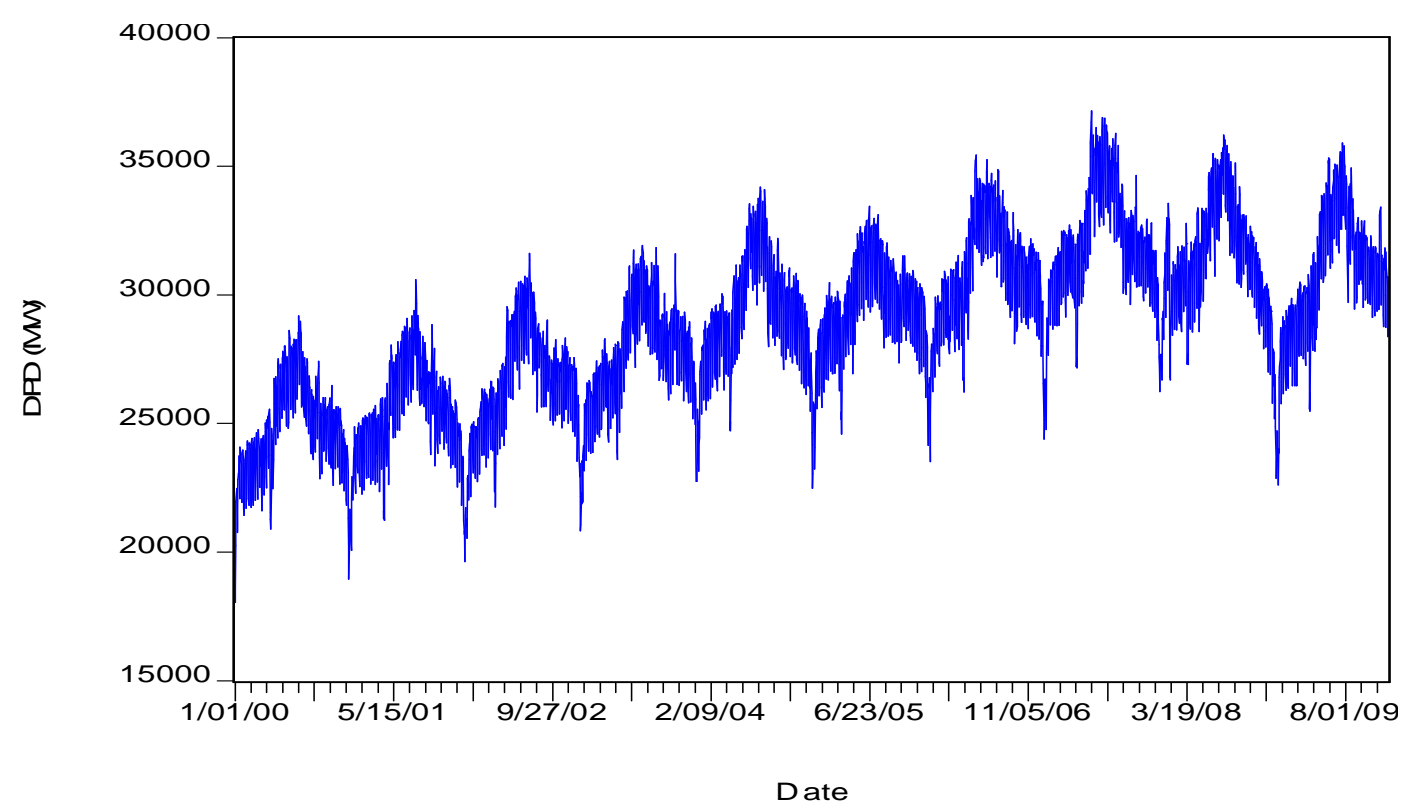

Figure 1. DPD (in megawatts) data from 1 January 2000 to 14 December 2009.

forecasting. Load forecasting using Bayesian statistics is discussed in literature. Chaturvedi (1996) discusses the use of robust Bayesian analysis. The author concludes that the use of robust Bayesian analysis overcomes the major drawback of coming up with a correct elicitation of a prior distribution. Interplay of Bayesian and frequentist analysis is discussed in Bayarri and Berger (2004). Bayarri and Berger (2004) identify the most common areas of important and useful connections between the Bayesians and frequentists as those scenarios when no external information, except the data and the model itself, is to be introduced into the analysis. Bayarri and Berger (2004) indicated that there are many areas of frequentist methodology that should be replaced by existing Bayesian methodology that provides superior answers. Other articles on the use of Bayesian statistics in electricity demand forecasting include those of (Jian et al., 2008; Kiartzia et al., 1997; Saini, 2008; Ohtsuka et al., 2010).

In this paper we develop a piecewise linear regression model. The focus in this paper in on the use of the Bayesian parameter estimates to a piecewise linear regression model in explaining the influence of temperature on daily peak electricity demand in South Africa. The paper concentrates on daily peak demand modelling which is important for providing short term forecasts which will assist in optimal dispatching of electrical energy. Our modelling approach is designed in such a way that we initially use a multivariate adaptive regression splines (MARS) algorithm to determine temperatures which separate the winter period from the weather neutral period and the temperature that separates the summer period from the weather neutral period. Estimation of the parameters of the model then follows using the least squares method. The next step involves the use of Bayesian statistics in developing posterior densities for the parameter estimates to the piecewise linear regression model. A Bayesian analysis provides a way of taking into account uncertainty in the estimation of the parameters. Uncertainty about the true values of the Bayesian parameter estimates is incorporated into the analysis through the use of a non-informative prior distribution. Arguably the Bayesian approach is more informative than the classical approach. This is important for load forecasters and system operators in the electricity sector as it helps in the determination of consistent and reliable supply schedules.

The rest of the paper is organized as follows; description of the load and temperature data; discussion based on models used in this paper; presentation of empirical results; detailed discussion of the results; conclusion.

\section{LOAD AND TEMPERATURE DATA}

Hourly data from Eskom, South Africa's power utility company is used in this study. Daily peak demand (DPD) data for the period 2000 to 2009 is used. We define DPD as the maximum hourly demand in a $24 \mathrm{~h}$ period which is a day. Figure 1 shows the graphical plot of daily peak demand and exhibits strong seasonality with a steep positive linear trend.

Figure 2 shows a graphical plot of daily demand profiles for the seven days of the week with DPD around20:00 h. Historical data on temperature were also 


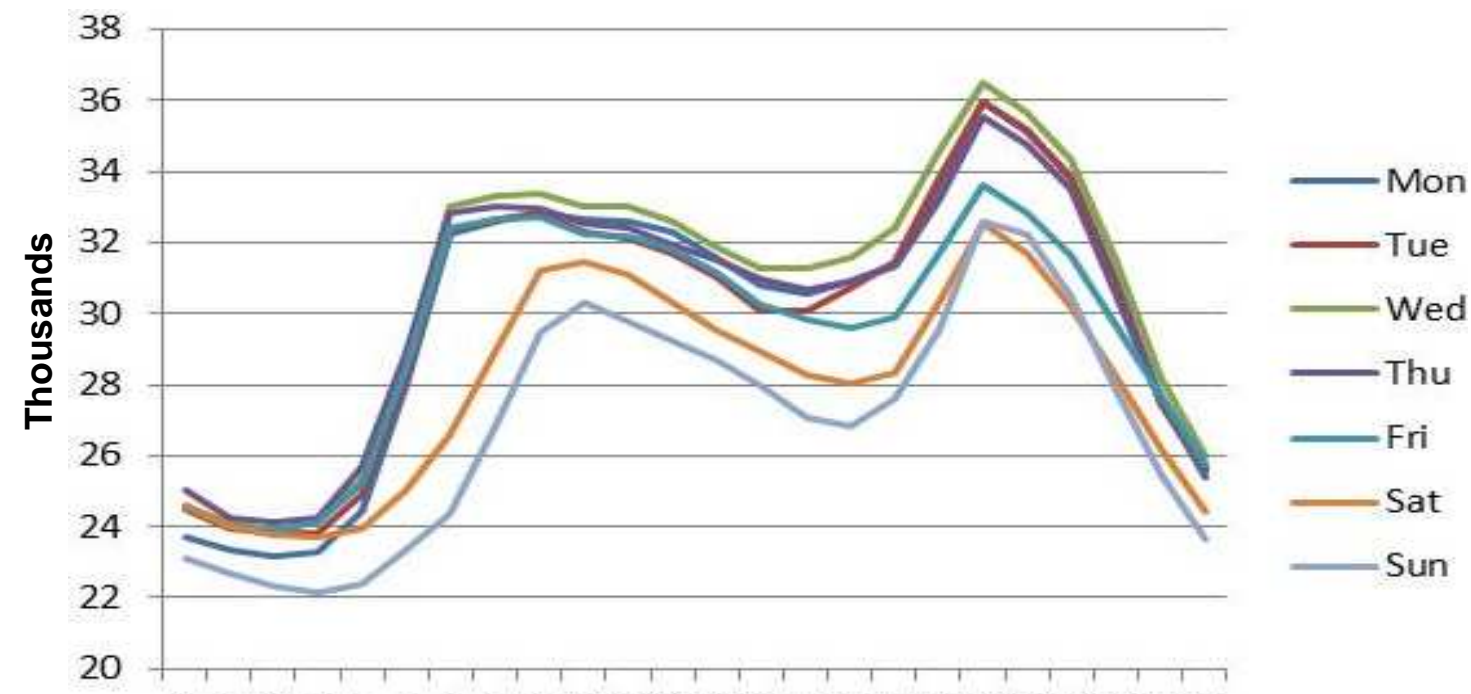

123456789101112131415161718192021222324

Figure 2. Typical daily load pattern. The $x$-axis shows time (hours) and $y$-axis demand (MW).

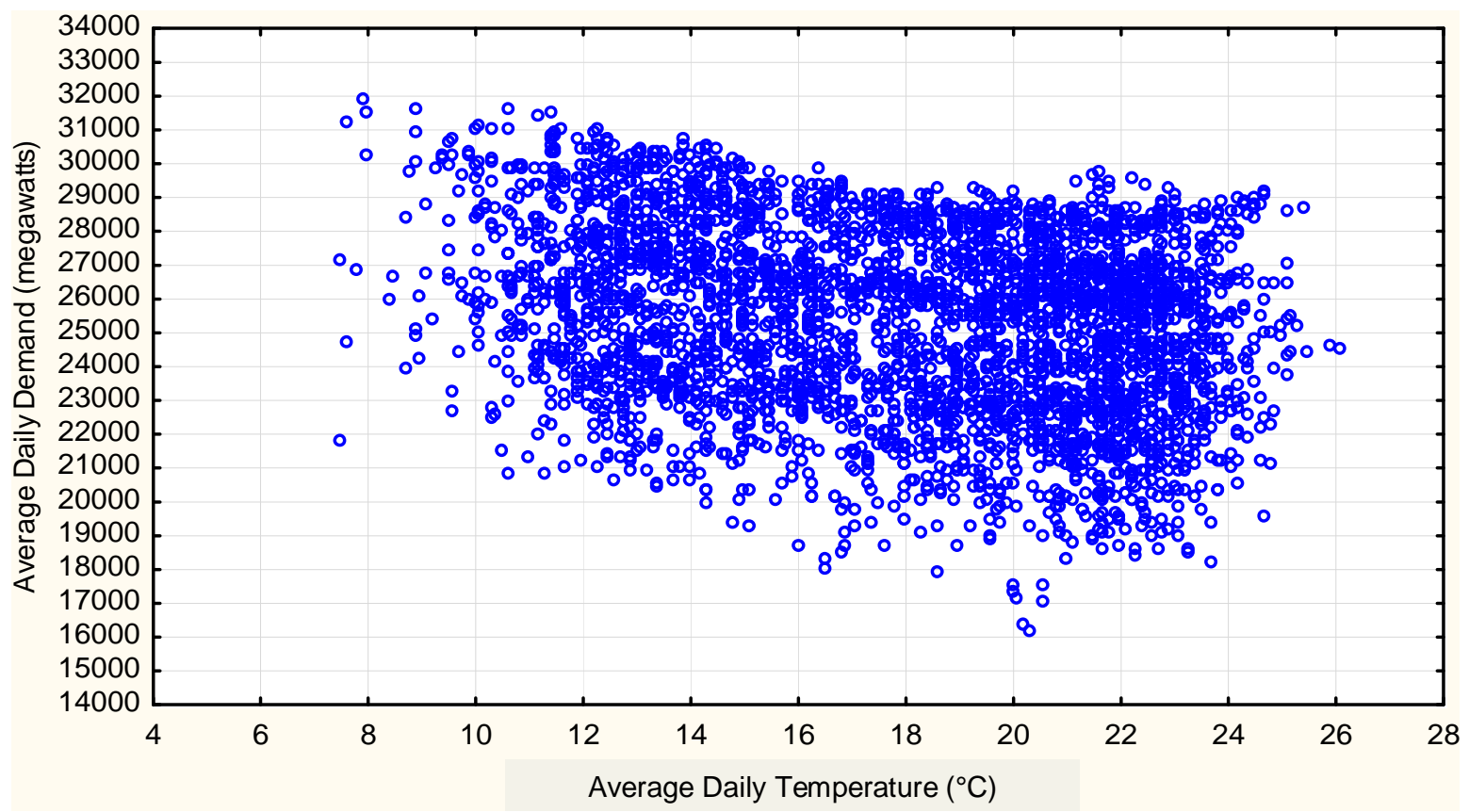

Figure 3. Scatter plot of average daily electricity demand (in megawatts) against average daily temperature $\left({ }^{\circ} \mathrm{C}\right)$ for the period $1 / 1 / 2000$ to $14 / 12 / 2009$.

collected from 22 meteorological stations from all the provinces of the country. The data were aggregated to obtain peak temperature corresponding to hour of daily peak demand, average daily maximum and minimum temperatures for the entire country.

Figure 3 shows a non-linear relationship between load and temperature. A visual inspection of Figure 3 shows that electricity demand is more sensitive to the winter period. The nonlinear relationship between load and temperature is modelled in literature using cooling degree-days and heating degree-days. Cooling degreedays (CDD) and the heating degree-days (HDD ) are 
estimated on the basis of the following two linear functions defined as:

$C D D=\max \left(T_{t}-T_{\eta v f}, 0\right)$

and

$H D D=\max \left(T_{r e f}-T_{t}, 0\right)$

where $T_{r}$ represents the temperature which separates the winter and summer periods of average daily electricity demand and temperature relationship and $T_{z}$ represents average daily temperature (mean outdoor air temperature) on dayt. If $T_{z}>T_{r \neq f}$ there are no HDD and similarly when $T_{t}<T_{\text {ref }}$ there are no CDD.

\section{THE MODELS}

Our modelling approach is in three steps. Initially a MARS algorithm is used in the determination of temperatures that separate winter and summer from the weather neutral period and also in selecting the important $\operatorname{knot}(\mathrm{s})_{T_{\text {ref }}}$, which represents the temperature which separates the winter and/or summer periods of daily peak electricity demand. The second step involves the development of a piecewise linear regression model and estimation of the parameters using the least squares method. In the third step Bayesian statistics is used in developing posterior densities for the parameter estimates to the piecewise linear regression model.

\section{The multivariate adaptive regression splines (MARS) frequentist model}

The general MARS model can be written as in Friedman (1991) and is given in Equation 1.

$f(x)=\varepsilon_{0}+\sum_{m=1}^{M} c_{m} s_{m}(x)$

where $B_{m}(x)$ is a basis function written as

$B_{m}(x)=\prod_{k=1}^{K_{\mathrm{m}}}\left[s_{k m}\left(x_{v(k m)}-t_{k m}\right)\right]$

$M$ is the number of basis functions, $c_{0}$ and $c_{m}$ are parameters, $K_{m}$ is the number of knots, $s_{\mathrm{km}}$ takes on values of either 1 or -1 and indicates the right or left sense of the associated step function, $v(k, m)$ is the label of the independent variable and $t_{k m}$ indicates the knot location.

A generalized cross validation (GCV) criterion is used to choose the best subset model using MARS. The MARS model discussed in this paper can be written as

$y_{t}=\beta_{0}+\beta_{1} \max \left(0, x_{p t}-t_{w}\right)+\beta_{2} \max \left(0, x_{p t}-t_{s}\right)$

where $y_{t}$ is (daily peak demand) DPD, $\beta_{0}, \beta_{1}$ and $\beta_{2}$ are the MARS model parameters with $\beta_{0}$ representing the weather neutral demand, $t_{w}$ denotes the temperature where the winter-sensitive portion of demand joins the non-weather-sensitive demand component, $t_{s}$ denotes the temperature where the summer-sensitive portion of demand joins the non-weather-sensitive demand component. Using the MARS algorithm the reference temperatures $\left(t_{w}\right.$ and $\left.t_{z}\right)$ were found to be approximately 18 and $2^{\circ} \mathrm{C}$ respectively, which is the weather neut ral period. Within this range of temperature values residents would neither use a heater nor a cooling system. The model identifies the winter sensitive, weather neutral and summer sensitive periods.

The MARS method does not make any assumptions about the functional relationship between the response variable and the predictor variables. The MARS modelling approach overcomes the major drawbacks of using artificial neural networks which have long training processes, interpretive difficulties and the inability to determine the relative importance of potential input variables. MARS has been used to solve high dimensional problems with complex model structures such as nonlinearities, interactions, multicollinearity and missing values (Chen, 1997; Friedman, 1991; De Gooijer and Ray, 2003; Tsekouras et al., 2007).

\section{Piecewise linear frequentist regression model}

The piecewise linear function used in this paper is shown in Equation 4.

$$
\begin{aligned}
& y_{\mathrm{z}}=\beta_{0}+\beta_{1} t+\beta_{2} X_{1}+\beta_{2} X_{2}+\sum_{\mathrm{d}=2}^{7} \alpha_{\mathrm{i}} D_{\mathrm{a}}+\sum_{j=2}^{12} \tau_{j} M_{j}+\mu H_{\mathrm{i}-1}+\delta H_{\mathrm{z}}+\gamma H_{\mathrm{z}+1}+\phi_{1} y_{\mathrm{i}-1} \\
& +\phi_{8} y_{z-3}+\phi_{6} y_{t-6}+\Phi_{7} y_{t-7}+\Phi_{31} y_{z-81}+\Phi_{178} y_{t-178}+s_{z}
\end{aligned}
$$

Where $y_{t}$ is DPD, $x_{1}=\left(x_{p t}-18\right) x_{1 t} \quad$ and $x_{1 t}=1$ if $x_{p t}<18$ and zero otherwise; $x_{2}-\left(x_{p t}-22\right) x_{2 t}$ and $x_{2 t}=1$ if $x_{p t}>22$ and zero elsewhere; $t$ is the trend component; $\varepsilon_{t}$ is the error term; $x_{p z}$ represents peak temperature (in degree celsius). The peak temperature 
is the temperature recorded at the hour of peak demand on day $t ; 18^{\circ} \mathrm{C}$ is the temperature to identify where the winter sensitive portion of demand join the non-weather sensitive demand component, $2^{\circ} \mathrm{C}$ is the temperature to identify where the summer sensitive portion of demand join the non-weather sensitive demand component, $\beta_{0}$ represents the mean daily peak demand observed in the non-weather sensitive period $18^{\circ} \mathrm{C} \leq \mathrm{x}_{\mathrm{pt}} \leq 22^{\circ} \mathrm{C}$. $H_{z}$, $H_{t-1}$ and $H_{z+1}$ are dummy variables representing holiday, day before and after a holiday respectively. The day of the week effect is represented by the dummy variable $D_{a}$, where $d$ represents the days Tuesday up to Sunday with Monday as the base period. $D_{d}$ equals 1 if day $d=$ Tuesday,..., Sunday and zero otherwise. The monthly effect is represented by $M_{j}$, where $j$ represents the months February up to December with January as the base month. $M_{j}$ equals 1 if month $j=$ February... December and zero otherwise. The lagged demand effects are modelled by $y_{t-1}, y_{t-3}, y_{t-6}, y_{t-7}, y_{t-31}, y_{t-175}$, where $y_{t-1}, y_{t-3}$ and $y_{t-6}$ will account for day to day variations in electricity peak demand. The week to week, monthly and semi-annual variations in electricity peak demand will be accounted for by $y_{t-7}, y_{t-31}$ and $y_{t-175}$ respectively.

\section{Bayesian linear regression model}

The advantage of the Bayesian approach lies in the fact that one can create the posterior distributions and hence can do inferences from the posterior distribution. From distributions of the parameters we are in a position to obtain quantiles, credible regions and perform other inferential tests. The methods can be generalized to more complicated situations. The normal multiple regression model is given by:

$y=X \beta+u$

Where $y=\left[Y_{1}, Y_{2}, \ldots, Y_{n}\right]^{\prime}$ is an $n \times 1$ vector of observations, $\quad X=\left[X_{0}, X_{1}, X_{2}, \ldots, X_{k-1}\right]$ is the $n \times k$ design matrix of observations on $k-1$ independent variables, $X_{0}=\left[\begin{array}{lll}1 & 1 & \ldots\end{array}\right]^{\prime}$ is an $n \times 1$ vector of ones. $\beta=\left[\beta_{0}, \beta_{1}, \ldots, \beta_{k-1}\right]^{\prime}$ is a $k \times 1$ vector of regression coefficients and $u$ is an $n \times 1$ vector of error terms. The error terms, $\boldsymbol{u}$ are assumed to be normally and independently distributed each with mean zero and common variance $\sigma^{2}$. The parameter vector is $\left(\boldsymbol{\beta}, \sigma^{2}\right)$. We then estimate $\beta$ and $\sigma^{2}$ together with their respective probability density functions (pdfs) and also test hypotheses involving the same parameter vector. In the frequentist approach the coefficient vector $\boldsymbol{\beta}$ is estimated by the ordinary least squares method using the MoorePenrose pseudo inverse, $\widetilde{\boldsymbol{\beta}}=\left(\boldsymbol{X}^{r} \boldsymbol{X}\right)^{-1} \boldsymbol{X}^{\prime} \boldsymbol{y}$. In the Bayesian approach the sample data is supplemented with additional information which is in the form of a prior distribution. Assuming that the random errors are independent and normally distributed we obtain the joint likelihood for $\boldsymbol{y}$ given $\boldsymbol{X}, \boldsymbol{\beta}$ and $\sigma^{2}$ as

$$
\begin{aligned}
& p\left(y \mid \boldsymbol{X}, \boldsymbol{\beta}, \sigma^{2}\right) \propto \frac{1}{\sigma^{n}} \exp \left[-\frac{1}{2 \sigma^{2}}(y-\boldsymbol{X} \boldsymbol{\beta})^{\prime}(\boldsymbol{y}-\boldsymbol{X} \boldsymbol{\beta})\right] \\
& \propto \frac{1}{\sigma^{n}} \exp \left\{-\frac{1}{2 \sigma^{2}}\left[(y-\hat{y})^{\prime}(\boldsymbol{y}-\hat{y})+(\boldsymbol{\beta}-\tilde{\boldsymbol{\beta}})^{\prime} \boldsymbol{X}^{\prime} \boldsymbol{X}(\boldsymbol{\beta}-\widehat{\boldsymbol{\beta}})\right]\right\} \\
& \propto \frac{1}{\sigma^{n}} \exp \left\{-\frac{1}{2 \sigma^{2}}\left[v s^{2}+(\boldsymbol{\beta}-\tilde{\boldsymbol{\beta}})^{\prime} \boldsymbol{X}^{\prime} \boldsymbol{X}(\boldsymbol{\beta}-\widehat{\boldsymbol{\beta}})\right]\right\}
\end{aligned}
$$

where $v=n-k_{s}$

$\widehat{\boldsymbol{\beta}}=\left(\boldsymbol{X}^{\prime} \boldsymbol{X}\right)^{-1} \boldsymbol{X}^{\prime} \boldsymbol{y}_{s} \hat{\boldsymbol{y}}=\boldsymbol{X} \widehat{\boldsymbol{\beta}}, s^{2}=\frac{(y-X \widehat{\beta})^{\prime}(\boldsymbol{y}-X \widehat{\beta})}{v}$ and

$\overline{\boldsymbol{\beta}} \mid \sigma^{2} N N\left(\boldsymbol{\beta}, \sigma^{2}\left(\boldsymbol{X}^{t} \boldsymbol{X}\right)^{-1}\right)$

In specifying our prior distribution we assume that our information is vague. We adopt the notation used in (Zellner, 1971). We use a non-informative Jeffreys' prior:

$p\left(\boldsymbol{\beta}, \sigma^{2}\right) \propto \frac{1}{\sigma^{2}},-\infty<\beta_{i}<\infty, i=1,2, \ldots, k_{i} 0<\sigma<\infty$.

Combining the prior distribution in (7) with the likelihood function in (6) we get the following joint posterior distribution

$$
\begin{aligned}
& p\left(\boldsymbol{\beta}_{r} \sigma^{2} \boldsymbol{y}_{r} \boldsymbol{X}\right)=p\left(y \mid \boldsymbol{X}_{i} \boldsymbol{\beta}_{r} \sigma^{2}\right) p\left(\boldsymbol{\beta}_{r} \sigma^{2}\right) \\
& p\left(\boldsymbol{\beta}, \sigma^{2} \mid y, \boldsymbol{X}\right) \propto \frac{1}{\sigma^{n+2}} \exp \left\{-\frac{1}{2 \sigma^{2}}\left[v s^{2}+(\boldsymbol{\beta}-\tilde{\boldsymbol{\beta}})^{\prime} \boldsymbol{X}^{\prime} \boldsymbol{X}(\boldsymbol{\beta}-\tilde{\boldsymbol{\beta}})\right]\right\}
\end{aligned}
$$

Integrating (8) with respect to $\sigma^{2}$ we get the following marginal posterior $p d f$ for the elements of $\boldsymbol{\beta}$ :

$p(\boldsymbol{\beta} \mid \boldsymbol{y}, \boldsymbol{X})=\int_{0}^{\infty} p\left(\boldsymbol{\beta}_{z} \sigma^{2} \mid \boldsymbol{y}, \boldsymbol{X}\right) d \sigma^{2}$

$\propto\left(v s^{2}+(\boldsymbol{\beta}-\widehat{\boldsymbol{\beta}})^{\prime} \boldsymbol{X}^{\prime} \boldsymbol{X}(\boldsymbol{\beta}-\widehat{\boldsymbol{\beta}})\right\}^{-n / 2}$ 


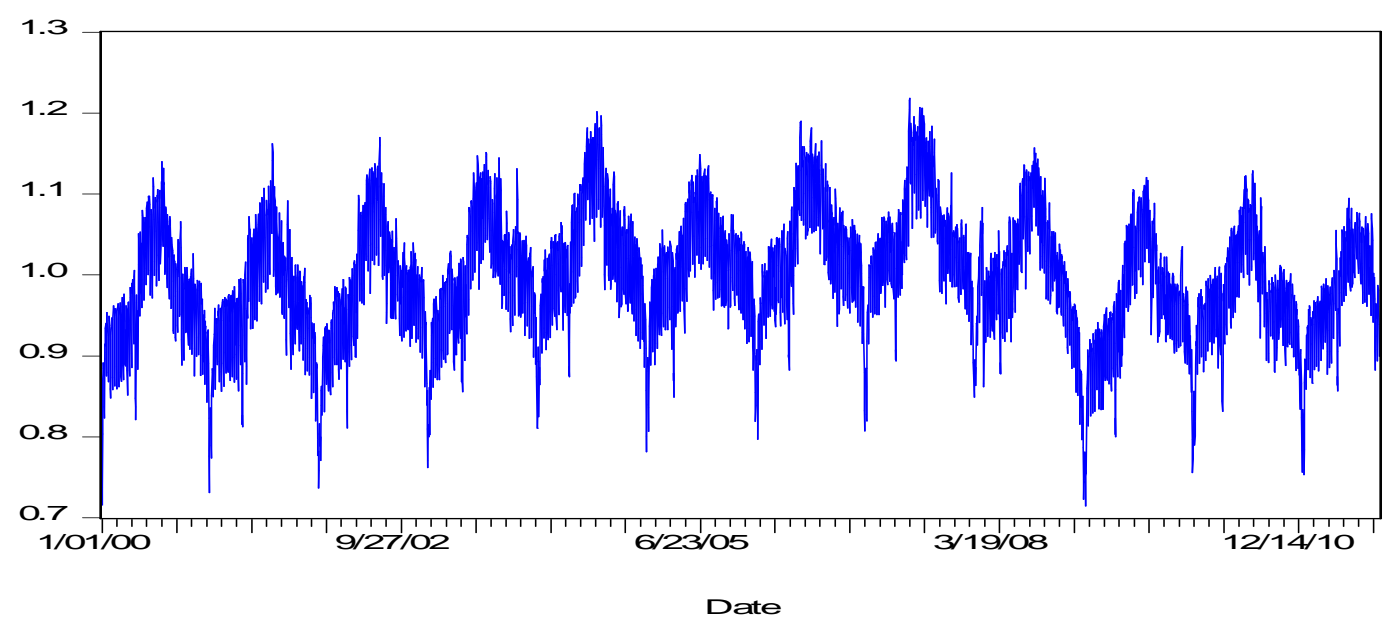

Figure 4. Daily peak demand after detrending.

where $\widehat{\beta}$ is the posterior mean vector given the data and prior information. The marginal posterior pdf in (9) follows a multivariate Student $t$ distribution. The marginal posterior distribution of $\beta_{i}$ follows a Student $t$ distribution, that is

$\frac{\beta_{i}-\hat{\beta}_{i}}{s\left(m^{i}\right)^{i / 2}} \cdots i_{v}$

where $m^{i i}$ is the $(i, i)^{\text {th }}$ element of $\left(\boldsymbol{X}^{i} \boldsymbol{X}\right)^{-1} \cdot \beta_{i}$ is the posterior mean of $\beta_{i}$ given the observed data and prior information. Now integrating (8) with respect to the elements of $\beta$ we get the marginal posterior $p d f$ of $\sigma^{2}$, that is

$n\left(\sigma^{2} \mid y_{x} X\right)=\int_{-\infty}^{\infty} \ldots \int_{-\infty}^{\infty} p\left(\boldsymbol{\beta}_{x} \sigma^{2} \mid \boldsymbol{y}, \boldsymbol{X}\right) d \boldsymbol{\beta}$

$\propto \frac{1}{c^{v+1}} \exp \left(-\frac{v s^{2}}{2 o^{2}}\right)$

The marginal posterior $p d f$ in (11) follows an inverse gamma $p d f$.

\section{EMPIRICAL RESULTS AND DISCUSSION}

Electricity demand is generally subject to seasonal changes and an upward positive linear trend. The graphical plot of DPD in Figure 1 exhibits strong seasonality with a steep positive linear trend. A visual inspection of the graphical plot of detrended DPD in Figure 4 shows that there has been a gradual decrease in annual peaks since 2007. This is probably due to energy efficiency and demand side management strategies put in place by Eskom, South Africa's power utility.

\section{Piecewise linear model}

The empirical results are presented in this area. Our piecewise linear regression model is given in Equation 12

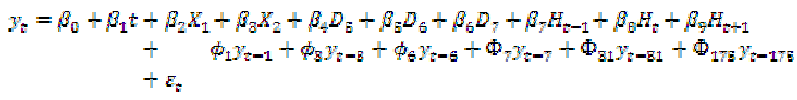

where all the variables are as defined in Equation 4. Substituting the coefficients of the variables we get

$$
\begin{gathered}
E\left(y_{t}\right)=25687.61+2.126 t-171.468\left(x_{n t}-18\right) x_{*}+24.85\left(x_{n t}-22\right) x_{2 t}-857.357 D_{5} \\
-2304.732 D_{6}-2564.954 D_{7}-779.485 H_{t-1}-1779.633 H_{t}-227.356 H_{t+1} \\
+\quad 0.797 y_{t-1}+0.0952 y_{t-3}+0.0783 y_{t-6}+0.192 y_{t-7} \\
-0.0346 y_{t-31}-0.0759 y_{t-178}
\end{gathered}
$$

The dummy variable $x_{1 t}$ is negative showing that if peak temperature decreases by one degree from $18^{\circ} \mathrm{C}$, electricity demand will increase by $171.468 \mathrm{MW}$. The coefficient of $x_{2 t}$ is positive showing that if temperature increases by one degree from $2^{\circ} \mathrm{C}$, electricity demand will increase by $24.85 \mathrm{MW}$. We conclude that electricity demand in South Africa is more sensitive to the winter period. All the coefficients of the dummy variables representing Friday, Saturday, Sunday, holiday, day before holiday and day after holiday are negative indicating that there is a decrease in demand during 
Table 1. In-sample forecasting evaluation for 01/01/2000 - 31-12-2009.

\begin{tabular}{lcc}
\hline \multirow{2}{*}{ Forecasting model } & \multicolumn{2}{c}{ Performance criteria (estimation period) } \\
\cline { 2 - 3 } & MAPE & RMSE \\
\hline Piecewise linear regression & 1.172 & 422.104 \\
\hline
\end{tabular}

Table 2. 99\% Bayesian credible intervals for the posterior $p d f s$ of the parameters.

\begin{tabular}{ccc}
\hline Parameter & 99\% Bayesian credible intervals & Posterior means of the parameters $\hat{\beta}_{i}$ \\
\hline$\beta_{0}$ & 23500 to 28000 & $E\left(\beta_{0}\right)=25687.61$ \\
$\beta_{1}$ & 1 to 3 & $E\left(\beta_{1}\right)=2.126$ \\
$\beta_{2}$ & -200 to -140 & $E\left(\beta_{2}\right)=-171.468$ \\
$\beta_{3}$ & -20 to 80 & $E\left(\beta_{3}\right)=24.85$ \\
$\beta_{4}$ & -960 to -820 & $E\left(\beta_{4}\right)=-897.357$ \\
$\beta_{5}$ & -2400 to -2200 & $E\left(\beta_{5}\right)=-2304.732$ \\
$\beta_{6}$ & -2640 to -2480 & $E\left(\beta_{6}\right)=-2564.954$ \\
$\beta_{7}$ & -925 to -650 & $E\left(\beta_{7}\right)=-779.485$ \\
$\beta_{8}$ & -1950 to -1650 & $E\left(\beta_{0}\right)=-1779.633$ \\
$\beta_{9}$ & -400 to -125 & $E\left(\beta_{9}\right)=-227.356$ \\
\hline
\end{tabular}

these periods. The largest decrease is on Sunday out of the three days of the week. Demand for electricity during holidays decreases significantly compared to a day before and after a holiday.

The root mean square error (RMSE) and mean absolute percentage error (MAPE) are generally used for evaluating the predictive power of short-term load forecasting (STLF) models (Munoz et al., 2010). These accuracy measures are used in the in-sample forecasting evaluation of the model. Several models were run and the results of the best model are summarized in Table 1. For the period $t=1, \ldots, n$, the RMSE, MAPE are given as:

$$
\begin{aligned}
& \text { RMSE }=\sqrt{\frac{1}{n} \sum_{t=1}^{n}\left(y_{t}-f_{t}\right)^{2},} \\
& M A P E=\frac{1}{n} \sum_{t=1}^{n} \frac{\left|y_{t}-f_{t}\right|}{y_{t}} 100,
\end{aligned}
$$

where $y_{t}$ and $f_{t}$ are the actual and predicted daily peak loads at time $t$.

\section{Posterior distributions of the parameters}

The posterior probability density functions ( $p d f \mathrm{~s}$ ) for the piecewise linear regression parameters are given in this area. We discuss the results of the parameters $\beta_{0}, \beta_{1}, \beta_{2}$ and $\beta_{3}$ and the rest of the posterior distributions of the parameters $\beta_{4}, \ldots, \beta_{9}$ are summarized in Table 2 and given in Figure 6.

The three demand-temperature lines for weekdays without the trend and holiday effects (that is, $\left(t=D_{5}=D_{6}=D_{7}=H_{t-1}=H_{t}=H_{t+1}=0\right)$ would be shown.

For the non-weather sensitive months $\left(18^{\circ} \leq x_{p t} \leq 22^{\circ}\right.$, $\mathrm{x}_{1 \mathrm{t}}=\mathrm{x} 2 \mathrm{t}^{0}$ ) we get $E\left(\beta_{0}\right)=\hat{\beta}_{0}=25687.61 \mathrm{MW}$ is the posterior mean daily peak electricity demand for the nonweather sensitive period. The posterior pdf for $\beta_{0}$ shown in Figure 5(a) shows the non-weather sensitive mean daily peak demand could take any value between 23500MW and $28000 \mathrm{MW}$.

There is a persistent increase in hourly electricity peak demand with time in South Africa. The slope is around 2 MW per year. It is highly unlikely that the slope exceeds 3 and highly unlikely that it is below 1 . The calculated mean 


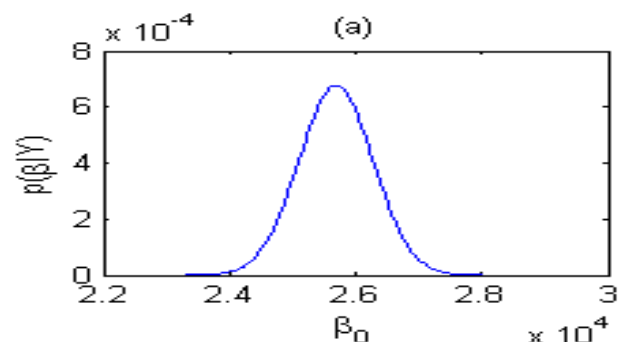

(c)

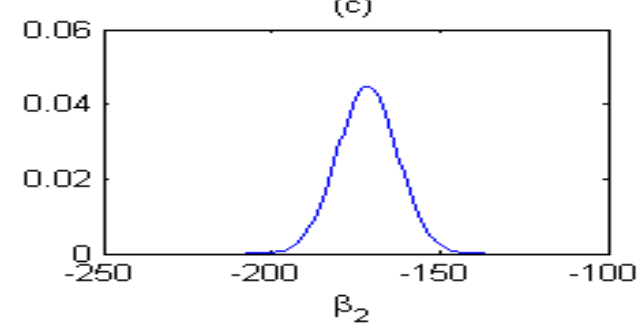

Figure 5. Posterior $p d f$ for parameters $\beta^{\prime} s$.
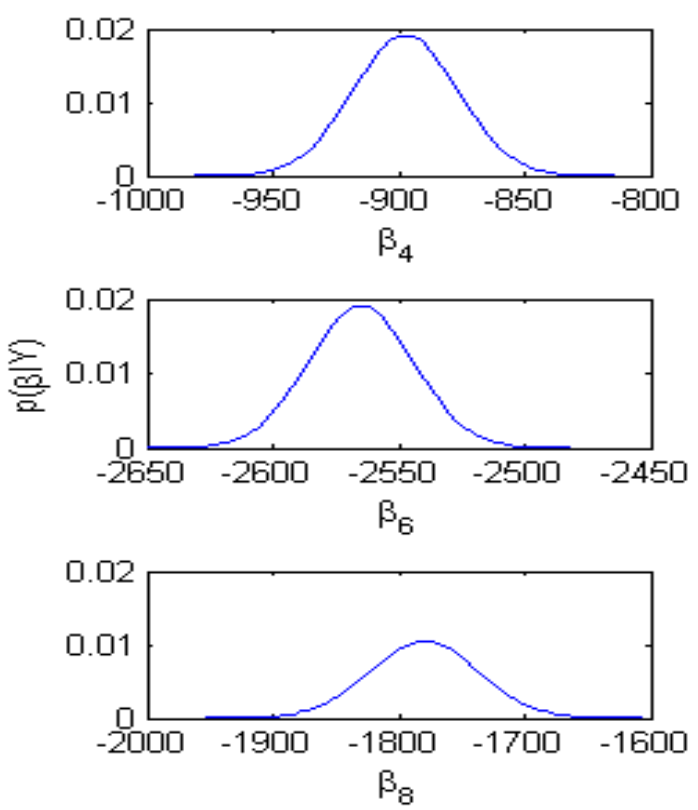

Figure 6. Posterior $p d f$ for parameters $\beta_{4}, \ldots, \beta_{9}$.

slope increase is $2.126 \mathrm{MW}$ per year. Figure $5(\mathrm{~b})$ shows the posterior $p d f$ for $\beta_{1}$.

For the winter sensitive months $\left(x_{\mathrm{pt}}<18, x_{1 t}-1, x_{2 t}-0\right)$ we get

$E\left(y_{z}\right)=2568761-171.468\left(x_{p z}-18\right) x_{2 z}$
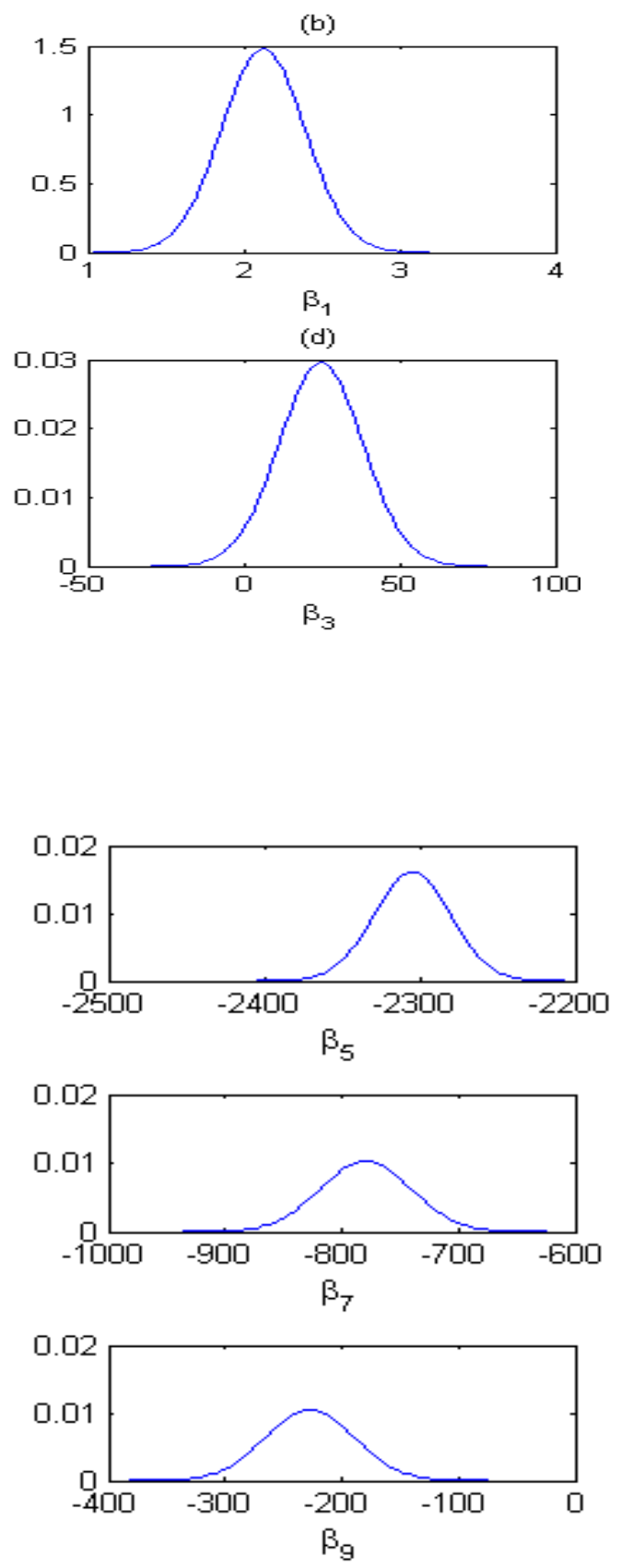

That is if temperature decreases by $1^{\circ} \mathrm{C}$ (from from 18 to $1^{\circ} \mathrm{C}$ ) the marginal increase in daily peak demand will be 171.468 MW. Because of the uncertainties' associated with demand, this increase could be any value between 140 and $200 \mathrm{MW}$. In other words, it is highly unlikely that this increase will be less than $140 \mathrm{MW}$ and highly unlikely that it will exceed 200 MW. Figure 5(c) shows the posterior pdf for $\beta_{2}$. 
For the summer sensitive months $\left(x_{p t}>22, x_{1 z}=0, x_{2 t}=1\right)$ we get

$E\left(y_{\hat{i}}\right)=25687.61+24.85\left(x_{p t}-22\right) x_{2 t}$

That is if temperature increases by $1^{\circ} \mathrm{C}$ (for example, from 22 to $23^{\circ} \mathrm{C}$ ) the marginal increase in demand wi ll be 24.85 MW. This marginal increase could be any value between -20 and $80 \mathrm{MW}$. The posterior pdf for parameter $\beta_{3}$ is shown in Figure $5(\mathrm{~d})$.

Table 2 summarizes the 99\% Bayesian Credible intervals of the posterior distributions of the parameters of the piecewise linear regression model.

\section{DISCUSSION}

A piecewise linear regression model was developed which related peak demand to temperature and the day of the week. This study has shown that aBayesian analysis provides a way of taking into account uncertainty in the estimation of the parameters. Uncertainty about the true values of the piecewise linear regression parameter estimates was incorporated into the analysis through the choice of a non-informative prior distribution.

Empirical results show that if temperature decreases by $1^{\circ} \mathrm{C}$ in the winter sensitive period (that is when temperature is less than $1^{\circ} \mathrm{C}$ ) then the average inc rease in daily electricity load will be $171.47 \mathrm{MW}$. This increase in electricity daily load ranges between 140 and $200 \mathrm{MW}$. Similarly if temperature increases by $1^{\circ} \mathrm{C}$ in the summer sensitive period (that is when temperature is greater than $2^{\circ} \mathrm{C}$ ) daily electricity load will increase by an av erage of $24.85 \mathrm{MW}$. This increase will range from -20 to $80 \mathrm{MW}$. These results show that demand of electricity is more sensitive to winter periods than summer periods in South Africa. This quantification of uncertainty about these parameters is important for load forecasters in Eskom as it helps them in the determination of consistent and reliable supply schedules. The developed model can either be used for predicting short-term daily peak demand or for weather normalization, which is estimating daily peak demand under normal weather conditions. The mean values of the parameters of the Bayesian approach are the same to the frequentist analogy because of the nature of the non-informative prior employed. It therefore seems that the frequentist properties of the Bayesian inferences of the model based on the prior are adequate. However the resultant posterior may be used in a way is not always available to frequentist.

\section{Conclusion}

The paper discussed the use of time series regression for modelling and predicting daily peak demand in South
Africa. The developed piecewise linear regression model captures demand drivers of electricity such as temperature, calendar effects and lagged demand. The model also accounts for residual correlation that may occur as a result of day to day, week to week, monthly and seasonal variations in electricity peak demand. This empirical study provides an extension of point forecasting to density forecasting so as to take into account uncertainty in the estimation of the parameters.

Areas for further study would include use of a full Bayesian MARS model in which we place a prior distribution on the whole MARS model space, treating the number of splines, knot points and all other parameters as unknown. Another interesting area of study would involve the use of robust Bayesian analysis and incorporation of the impact of energy efficiency and demand side management strategies being put in place by Eskom, South Africa's power utility. These areas will be studied in our future research.

\section{ACKNOWLEDGEMENTS}

The authors are grateful to Eskom for providing the data and to the anonymous reviewers for their comments which have resulted in a significant improvement of this paper.

\section{REFERENCES}

Bayarri MJ, Berger JO (2004). The interplay of Bayesian and frequentist analysis. Stat. Sci. 19(1):58-80.

Chaturvedi A (1996). Robust Bayesian analysis of the linear regression model. J. Stat. Plan. Infer. 50:175-186.

Chen LA (1997). Multivariate regression splines. Comput. Stat. Data Anal. 26:71-82.

Cottet R, Smith M (2003). Bayesian modeling and forecasting of intraday electricity load. J. Am. Stat. Assoc. 11:839-849.

De Gooijer JG, Ray BK (2003). Modelling vector nonlinear time series using POLYMARS. Comput. Stat. Data Anal. 42:73-90.

Fan S, Hyndman RJ (2011). Short-term load forecasting based on a semi-parametric additive model, IEEE Transactions on Power Systems 26(3).

Friedman JH (1991). Multivariate adaptive regression splines. Annals Stat. 19(1):1-141.

Hekkenberg M, Moll HC, Schoot AJM (2009). Dynamic temperature dependence patterns in future energy demand models in the context of climate change. Energy 34:1797-1806.

Jian C, Jue G, Hu L (2008). Forecasting energy demand of China using Bayesian combination model. China Popul. Resour. Environ. 18(4):50-55.

Kiartzia S, KehagiasA, BarkirtzisA, Petridis V (1997). Short term load forecasting using a Bayesian combination method. Electr. Power Energy Syst. 19(3):171-177.

Mirasgedis S, Sarafidis Y, Georgopoulou E, Lalas DP, Mschovitis M, Karagiannis F, Papakonstantinou D (2006). Models for mid-term electricity demand forecasting incorporating weather influences. Energy 31:208-227.

Munoz A, Sanchez-Ubeda EF, Cruz A, Marin J (2010). Short-term forecasting in power sytems: A guided tour. Energy Syst. 2:129-160.

Ohtsuka Y, Oga T, Kakamu K (2010). Forecasting electricity demand in Japan: A Bayesian spatial autoregressive ARMA approach. Comput. Stat. Data Anal. 54:2721-2735.

Saini LM (2008). Peak load forecasting using Bayesian regularization, 
resilient and adaptive back propagation learning based artificial neural networks. Electric Power Syst. Res. 78:1302-1310.

Taylor JW (2006). Density forecasting for the efficient balancing of the generation and consumption of electricity. Int. J. Forecast. 22:707724.

Tsekouras GJ, Dialyna EN, Hatziargyriou ND, Kavatza S (2007). A non-linear multivariable regression model for midterm energy forecasting of power systems. Electric Power Syst. Res. 77:15601568.
Zellner A (1971). An introduction to Bayesian inference in econometrics. (1st edition) New York, John Wiley \& Sons. 